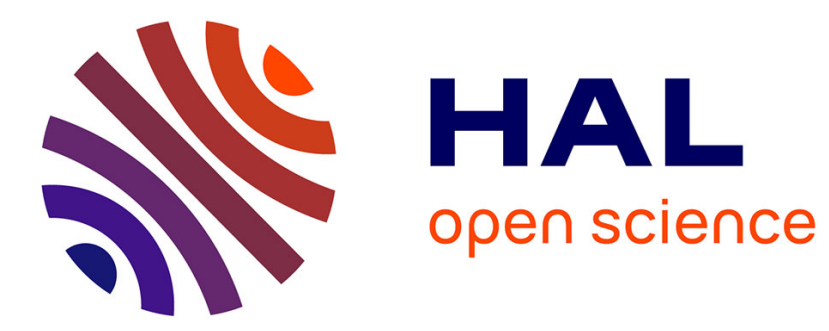

\title{
AUTOMATIC NEEDLE LOCALIZATION IN 3D ULTRASOUND IMAGES FOR BRACHYTHERAPY
}

\author{
Hatem Younes, Sandrine Voros, Jocelyne Troccaz
}

\section{To cite this version:}

Hatem Younes, Sandrine Voros, Jocelyne Troccaz. AUTOMATIC NEEDLE LOCALIZATION IN 3D ULTRASOUND IMAGES FOR BRACHYTHERAPY. International Symposium on Biomedical Imaging, ISBI'2018, Apr 2018, Washington, United States. pp.1203-1207. hal-01800108

\section{HAL Id: hal-01800108 \\ https://hal.science/hal-01800108}

Submitted on 25 May 2018

HAL is a multi-disciplinary open access archive for the deposit and dissemination of scientific research documents, whether they are published or not. The documents may come from teaching and research institutions in France or abroad, or from public or private research centers.
L'archive ouverte pluridisciplinaire HAL, est destinée au dépôt et à la diffusion de documents scientifiques de niveau recherche, publiés ou non, émanant des établissements d'enseignement et de recherche français ou étrangers, des laboratoires publics ou privés. 


\title{
AUTOMATIC NEEDLE LOCALIZATION IN 3D ULTRASOUND IMAGES FOR BRACHYTHERAPY
}

\author{
Hatem YOUNES, Sandrine VOROS and Jocelyne TROCCAZ \\ Univ. Grenoble Alpes, CNRS, CHU Grenoble Alpes, Grenoble INP, \\ INSERM, TIMC-IMAG, F-38000 Grenoble, France
}

\begin{abstract}
Needle segmentation in ultrasound images may be the indispensable step to solve other problems, such as the detection of radioactive seeds in ultrasound images for brachytherapy treatment. In this paper we propose a novel method to localize accurately curved paths of flexible needles in threedimensional (3D) ultrasound images. Our method is based on an automatic thresholding step where the bayesian classifier theory is applied to select a needle's voxels from the background. The next step consists in detecting the needle and representing its curvature. Here we propose to fit a parametric polynomial of low degree to representing the parabolic curvature. We describe a first evaluation on a data set of 9 patients.
\end{abstract}

Index Terms - Needle segmentation, 3D Ultrasound image, Bayesian classifier, Prostate brachytherapy.

\section{Introduction}

Prostate cancer is the second most frequently diagnosed cancer in men worldwide, with 1.1 million new cases in 2012 [1]. In Europe, prostate cancer is the first leading type of cancer in terms of new cases and the third leading cause of cancer death among males [2]. In 2012 the highest incidence rates were estimated in Northern and Western European countries such as Norway (193 per 100,000) and France (187 per 100,000) [2]. Among the wide range of possible treatments[3], brachytherapy with permanent seeds (also called low-dose brachytherapy) is considered an effective treatment modality for low risk prostate cancer with low urinary toxicity[4].

Physicians use brachytherapy needles to implant seeds into the patient's prostate. Parallel needles are inserted through a grid named "template". Needles insertion and seeds placement are performed under the control of ultrasound images -most often a set of parallel axial images (figure1) resulting in a reconstructed 3D volume-. Because brachytherapy aims at delivering a specific and homogeneous radiation dose to the prostate whilst limiting the dose delivered to organ at risks (urethra, rectum and bladder), it is based on a planning phase determining the optimal placement of seeds. Due to the limited precision of seed placement, planning generally needs to be updated during the procedure. In the perspective of an automated process, needle position monitoring is a mandatory preliminary stage. Furthermore needles are frequently used in other interventional procedures such as biopsy and their automatic detection is of primary interest. In this paper we propose a novel method for automatic needle segmentation in 3D ultrasound images. This modality, widely used because of its non-invasiveness, its ability to visualize moving soft tissue in real-time and its low-cost, suffers from intrinsic limitations: noise, artifacts-due to the anatomy or to needles for instance-, signal attenuation with depth and high variability depending on the user and the patient. All these reasons make the needle detection challenging.

Several approaches have been proposed for segmentation and localization of needles in 3D ultrasound images. Novotny et al. [6] proposed to apply the PCA algorithm method on the clusters of thresholded voxels and to identify the needle as the longest line on the brightest cluster. Hough Transform (HT) was also used to localized straight needles, Zhou et al. [7] proposed two improvements of the classical HT by introducing $3 \mathrm{D}$ and randomizing aspects. It is important to underline that although needles are initially straight, they can bend during insertion in the living tissue. Ding et al. [8] proposed to segment the needle in two orthogonal planes. This method was developed by Aboofazeli et al. [9] to detect a curved needle. They used a ray casting process to obtain projected 2D images. Ayvaci et al.[10] first proposed machine learning techniques to detect a biopsy needle. They minimized an energy function from MRI/TRUS fusion guided biopsy videos. Zhao et al. [11] proposed a comparison study of biopsy needle localization methods in 3D ultrasound images.

Most methods start with a binarization step which consists in classifying voxels into two classes given their intensity. Voxels with level grey higher than a threshold $\tau$ belong to the needle whilst the others correspond to the organs. Since all further steps process the binarized images, the choice of $\tau$ has a very high impact on the segmentation performance. As mentioned previously, this choice is made difficult due to high variability depending on the patient and ultrasound acquisition parameters tuning. $\tau$ might even vary in a single image due to signal attenuation. We thus propose to apply a bayesian 
classifier to obtain thresholded voxels (section 2.1) for improved accuracy and robustness to image variability. Then, the needle is detected among the thresholded voxels as a spatial parametric polynomial curve fitting (section 2.2). Results are described in section 3 .

\section{Methods}

The proposed method processes each needle separately by considering a region of interest (ROI) constructed around the planned trajectory of the needle. The insertion direction in the template allows defining a parallelepipedic bounding box where the needle has to be searched for.

\subsection{Thresholding}

A set of voxels $X$, with intensity $I(X) \subseteq \mathbb{R}$ and their coordinates $X \subseteq \mathbb{R}^{3}$ is divided into binary groups: needle voxels $\left(X_{n}\right)$ and background voxels $\left(X_{b}\right)$.

$$
\begin{aligned}
& X_{n}=\{x \in X: I(x) \geq \tau\} \\
& X_{b}=\{x \in X: I(x)<\tau\}
\end{aligned}
$$

To define $\tau$ automatically for various data sets, several authors proposed to use histogram value. For example Beigi et al. [12] chose the threshold value based on the cumulative sum of the number of pixels. Ultrasound image contains bright artifacts which intensities may be greater than needle intensities, resulting in wrong classification of these voxels. To solve this problem, authors used speckle reduction techniques, which are high complexity procedures. So we propose to apply instead a Bayesian classifier to accomplish thresholding without any preprocessing step.

\subsubsection{Gaussian mixture model}

Histogram value (figure 2) shows the existence of two separated peaks which describe the background and the needle. The histogram can be modeled using an additive Gaussian mixture model:

$$
P(y)=\sum_{i=1}^{M} \pi_{X_{i}} p_{i}(y)
$$

Where $y=I(x)$ is the intensity of $x \in X, M$ is the number of mixed components, $\pi_{X_{i}}$ is the coefficient of $i^{t h}$ component and $p_{i}(y)=\frac{1}{\sqrt{2 \pi} \sigma_{i}} e^{-\frac{\left(y-\mu_{i}\right)^{2}}{2 \sigma_{i}^{2}}}$ is the density that defines the $i^{\text {th }}$ component. To select the needle peak we use a Bayesian classifier where we assume that there are exactly two classes.

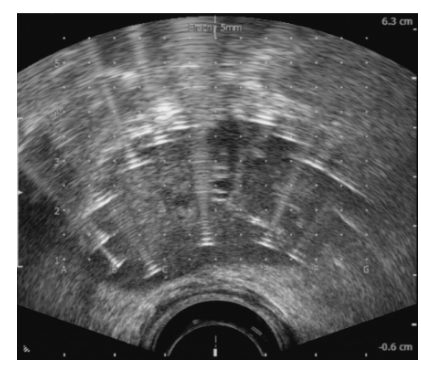

Fig. 1: 2D axial image, the needles perpendicular to the slice are figured as the points radiating linear artifacts

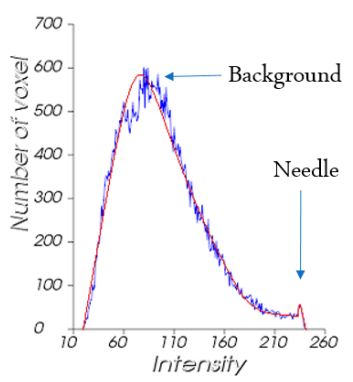

Fig. 2: Typical histogram in a bounding box with needle and background peaks

\subsubsection{Naive Bayes classification}

Bayesian classifiers assign the most likely class to a given observed feature [13]:

$$
C_{\text {Bayes }}(y)=\underset{X_{i}}{\operatorname{argmax}} P\left(C l=X_{i} \mid I(x)=y\right)
$$

Where $C l=\left\{X_{n}\right.$ (Needle), $X_{b}$ (Background) $\}$.

Naive Bayes classifier assumes that the intensities $I(X)$ are independent within each class, so the posterior probability of each class is calculable as follows based on Bayes theorem:

$$
P\left(C l=X_{i} \mid I(x)=y\right)=\frac{\pi_{X_{i}} P\left(I(x)=y \mid C l=X_{i}\right)}{P(I(x)=y)}
$$

Therefore, in the case of binary classification, naive Bayesian classifier assigns to each voxel the class which maximizes

$$
\underset{X_{i}}{\operatorname{argmax}}\left\{\frac{\pi_{X_{n}} P\left(I(x)=y \mid C l=X_{n}\right)}{P(I(x)=y)}, \frac{\pi_{X_{b}} P\left(I(x)=y \mid C l=X_{b}\right)}{P(I(x)=y)}\right\} .
$$

So naive Bayesian classification is summarized by the followed inequalities :

$$
\pi_{X_{n}} P\left(I(x)=y \mid C l=X_{n}\right) \underset{X_{b}}{\stackrel{X_{n}}{\gtrless}} \pi_{X_{b}} P\left(I(x)=y \mid C l=X_{b}\right)
$$

Voxel $x \in X$ is classified in $X_{n}$ if the posterior probability of $X_{n}$ is larger than $X_{b}$. The application of this probabilistic model requires the knowledge of several parameters which vary from image to another, or even from one needle ROI to another in the same image. These parameters are optimized according to a criterion of maximum likelihood to approach as much as possible the sought distribution. This procedure is often done iteratively via the expectation-maximization algorithm (EM [14]) explained as follows.

The aim of EM is to estimate the unknown parameters representing the mixing value between the Gaussians (the means, the variances and the prior probabilities) which maximize the posterior probability given an observed feature. EM algorithm is a dedicated iterative process, which computes 
the maximum-likelihood of an observed vector of features for each iteration. Jeff A. Bilmes [15] developed in his tutorial the procedure of the EM parameter estimation.

$$
\begin{array}{r}
\pi_{X_{i}}^{n e w}=\frac{1}{N} \sum_{j=1}^{N} p\left(X_{i} \mid x_{j}, \theta^{\ell}\right) \\
\mu_{X_{i}}^{n e w}=\frac{\sum_{j=1}^{N} x_{j} p\left(X_{i} \mid x_{j}, \theta^{\ell}\right)}{\sum_{j=1}^{N} p\left(X_{i} \mid x_{j}, \theta^{\ell}\right)} \\
\sigma_{X_{i}}^{\text {new }}=\frac{\sum_{j=1}^{N} x_{j} p\left(X_{i} \mid x_{j}, \theta^{\ell}\right)\left(x_{j}-\mu_{X_{i}}^{n e w}\right)^{2}}{\sum_{j=1}^{N} p\left(X_{i} \mid x_{j}, \theta^{\ell}\right)}
\end{array}
$$

Where $N$ is the number of voxels within the ROI and $\theta^{\ell}$ is the vector of parameters to be estimated at the $\ell^{\text {th }}$ iteration.

The algorithm proceeds by using the newly derived parameters, calculated as equations (6), as the guess for the next iteration until convergence. EM typically converges to a local optimum estimated when parameters values stabilize:

$\left|\theta^{\ell}-\theta^{\ell+1}\right|<\epsilon$. While EM converges to a local optimum, it requires a meaningful initialization.

We want the means $\left(\mu_{X_{n}}, \mu_{X_{b}}\right)$ to converge to the values of peaks corresponding to the needle and the background classes. Histogram of figure 2 shows us that the background class is the dominant one, so any initialization value of $\mu_{X_{i}}$ between the peaks values will converge to the background peak. The best initialization value of $\mu_{X_{n}}$ to reach the needle peak is 255. Any initialization of $\mu_{X_{b}}$ between peaks values $[120,210]$ converges to the background peak. Furthermore we choose the initialization values of the variances $\left(\sigma_{X_{n}}, \sigma_{X_{b}}\right)$ to be sure that EM remains able to converge to the values of peaks of the two classes. We selected $\left(\sigma_{X_{n}}, \sigma_{X_{b}}\right)=(7,60)$. These values are drawn from the variance values observed on histograms of bounding boxes of the first image and were robust when applied to the other images. Finally prior probability of the needle class $\pi_{X_{n}}$ is drawn from the number of voxels which correspond to the needle within a boundary box: $\left(\pi_{X_{n}}, \pi_{X_{b}}\right)=\left(0.03,1-\pi_{X_{n}}\right)$. Using EM algorithm we are able to estimate parameters required to construct naive Bayesian classifier. Therefore, needle voxels are identified from needle volume (left of figure 3 ), and the point cloud corresponding to the thresholded needle is shown in the right of figure 3 , when inequality (5) is satisfied.

\subsection{Needle Localization}

The second stage consists in localizing the needle within the thresholded voxels selected by the naive Bayesian classification. Most tool localization methods in thresholded 3D ultrasound images are based on Hough Transform(HT) for straight needle [7], and on the model-fitting approach [17],[16] for curved tool. Random sample consensus (RANSAC) algorithm [18] is used to compute the parameters of the model
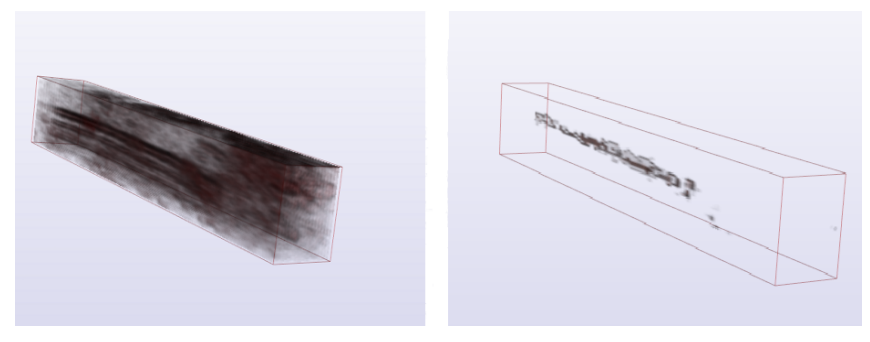

Fig. 3: A 3D needle volume (left) before and (right) after thresholding step

fitting which makes the procedure costly. We chose to use model fitting to localize the curved needle among the thresholded voxels, and limit candidate crossing points for parameters calculation to still have a real time results.

\subsubsection{Parametric curve}

The needle axis is represented by a spatial parametric polynomial curve $c(t ; \mathbf{H}): \mathbb{R} \longrightarrow \mathbb{R}^{3}$ of low order $n-1$ :

$c(t ; \mathbf{H})=\left(\begin{array}{c}h_{11}, \ldots, h_{1 n} \\ h_{21}, \ldots, h_{2 n} \\ h_{31}, \ldots, h_{3 n}\end{array}\right)\left(\begin{array}{c}1 \\ t \\ : \\ t^{n-1}\end{array}\right), \quad t \in \mathbb{R}$

where $\mathbf{H}=h_{i, j}$ is a matrix of the curve's coefficients and $t$ is a curve parameter. We use $n=3$ to model needles which exhibit a parabolic curvature. So the matrix $\mathbf{H}$ is found by solving the system of linear equations as follows:

$$
c\left(t_{i} ; \mathbf{H}\right)=p_{i}, \quad 1 \leq i \leq 3
$$

Where $p_{i} \in \mathbb{R}^{3}$ are the 3 control points through which the curve is required to pass and $t_{i}=\frac{\left(p_{i}-p_{1}\right) k_{0}}{|| k_{0} \|}$ an estimate of the projection of $p_{i}$ onto the curve as the principal direction $k_{0}$ of point cloud. For each possible triplet of control points, both the matrix of coefficients $\mathbf{H}$ and its $\operatorname{cost} C$ are calculated. $C$ corresponds to the distance between the point cloud and the curve.

$$
C(\mathbf{H})=\sum_{x \in X_{n}} d(x ; \mathbf{H})^{2}
$$

where $d(x ; \mathbf{H})$ is the distance between point $x$ and the curve approximated as $\|x-c(t ; \mathbf{H})\|$, where $t$ is the projection of $x$ onto the curve. The curve $c\left(t ; \mathbf{H}^{*}\right)$ that minimizes $C(\mathbf{H})$ is selected as the model that best approximates the needle.

$$
\mathbf{H}^{*}=\underset{\mathbf{H}}{\operatorname{argmin}} C(\mathbf{H})
$$

We propose to generate all the triplets of points $\left(p_{1}, p_{2}, p_{3}\right)$ from three regions $\left(X_{1 n}, X_{2 n}, X_{3 n}\right) \subset X_{n}$. These regions are drawn from the cloud of voxels $X_{n}$ where $X_{1 n}, X_{2 n}, X_{3 n}$ correspond to the beginning, middle and end of the detected voxels in the needle direction (figure 5). 

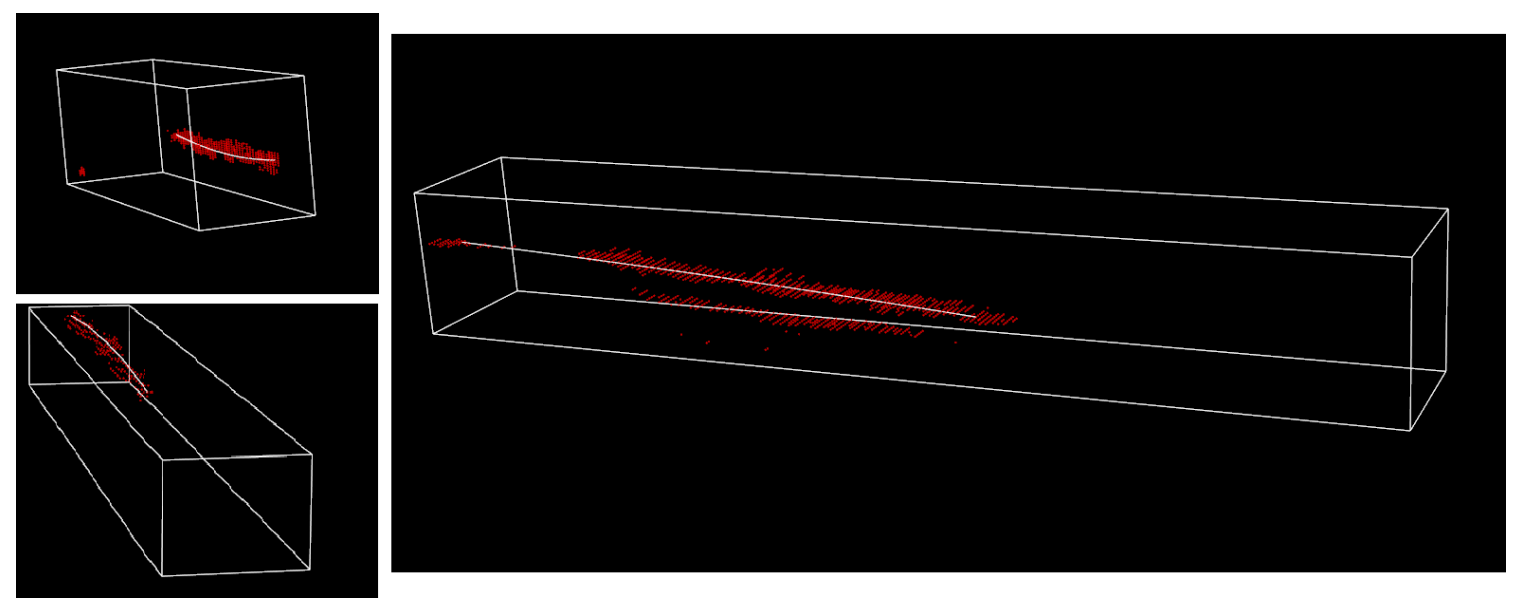

Fig. 4: Results: (left) two needles completely visible, (right) needle containing an occluded region

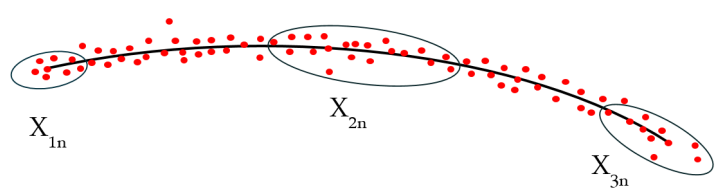

Fig. 5: The regions $\left(X_{1 n}, X_{2 n}, X_{3 n}\right)$ used to select the triplet control points $\left(p_{1}, p_{2}, p_{3}\right)$

\section{Results}

The algorithm was implemented in CamiTK which is an open source, cross platform generic tool, written in C++[19]. It was tested on anonymized 3D ultrasound images of 9 real patients. The image size is about $765 \times 575 \times 65$. The bounding box size is about $50 \times 50 \times 65$. The size of a voxel is $0.26 \times 0.26 \times 1$. The number of needles per image is 14 .

We evaluated the results of the implemented algorithm using a manual needle segmentation. Thus, we have firstly measured the objectivity of our manual segmentation by repeatedly segmenting two arbitrary needles in two different images, at distant time. The standard deviations obtained were $\left\{\right.$ tip $<0.5 \mathrm{~mm}$, axis $\left.<1^{\circ}\right\}$.

Images of real patients may contain some needles not fully detectable, for instance, when crossing a shadowed region (figure 4). Table 1 provides the number of needles per category (fully/partially visible) in each image of the data set. Error in the tip localization for fully visible needles is in $[0.6 ; 2.7] \mathrm{mm}$ with a mean value of $1.4 \mathrm{~mm}$, and error in the needle axis is in $\left[0.5^{\circ} ; 3.5^{\circ}\right]$ with a mean value of $1.5^{\circ}$. Error in the tip localization for partially visible needles is in $[2 ; 8] \mathrm{mm}$ with a mean value of $4.2 \mathrm{~mm}$, and error in the needle axis is in $\left[2^{\circ} ; 7^{\circ}\right]$ with a mean value of $6^{\circ}$. The average needle length being $45 \mathrm{~mm}$; the error in the tip localization is about $3 \%$ of the needle length for fully visible needles and about 9.3\% for partially visible needles. Each needle detection
Table 1: Number of fully/partially visible needle per image

\begin{tabular}{ccc}
\hline Patient & $\begin{array}{l}\text { Number of needles } \\
\text { fully visible }\end{array}$ & $\begin{array}{l}\text { Number of needles } \\
\text { partially visible }\end{array}$ \\
\hline 1 to 7 & 11 & 3 \\
\hline 8 & 5 & 9 \\
\hline 9 & 7 & 7 \\
\hline
\end{tabular}

takes about $1.25 \mathrm{~s}$ (un-optimized program implemented on a system with a $3.50-\mathrm{GHz}$ processor and $32 \mathrm{~GB}$ of RAM). The EM algorithm was able to converge on all the 126 needles localized showing the robustness of the needle peak detection.

\section{Conclusion}

We have proposed a novel technique to detect curved needles without any pre-processing. However, information from the planning of needle position could allow to optimize the size of the region of the bounding box. We used naive Bayesian classifier to select needle voxels among volume voxels. Localization of the needle within selected voxels is done by spatial parametric polynomial curve of low order. The proposed algorithm was tested on realistic images of patients. Needle path would be excellent prior information to localize seeds, it will be our next objective.

\section{Acknowledgements}

We thank LATIM and Brest University hospital for giving access to patient images. This work was partly supported by the French ANR within the Investissements dAvenir program (Labex CAMI ANR-11-LABX) and through the FOCUS project (ANR-16-CE19-0011). 


\section{References}

[1] Lindsey A. Torre, Freddie Bray, Robecca L. Siegel, Jacques Ferlay, Joannie Lortet-Tieulent, Ahmedin Jemal, " Global Cancer Statistics" A Cancer Journal for Clinicians (2012); p.69:90

[2] J. Ferlay, E. Steliarova-Foucher, J. Lortet-Tieulent, S. Rosso, J.W.W. Coebergh, H. Comber, D. Forman, F. Bray, "Cancer incidence and mortality patterns in Europe: Estimates for 40 countries in 2012" European Journal of Cancer (2013); 49: p. 1374: 1403.

[3] Richard.E., Peschel and Jhon W Colberg. "Surgery, brachytherapy, and external-beam radiotherapy for early prostate cancer" The lancet oncology (2003); 4.4: p. 233: 241

[4] Raziee, H., Berlin, A., Chung, P. W., Helou, J. A., Jiang, H., Crook, J. M. and Catton, C. N. "Permanent seed brachytherapy for low risk prostate cancer, long term outcome, and urinary toxicity". American Society of Clinical Oncology (2017); 99: p. E262

[5] Jinlan Huang, John K. Triedman, Nikolay V. Vasilyev, Yoshihiro Suematsu, Robin O. Cleveland, Pierre E. Dupont, "Imaging Artifacts of Medical Instruments in Ultrasound-Guided Interventions". Journal of Ultrasound in Medical (2007); 26: p. 1303: 1322

[6] Novotny, P., J. Cannon, and R. Howe, "Tool localization in 3D ultrasound images". Med. Image Comput. Comput. Assisted Interven.(MICCAI) (2003); p. 969:970.

[7] H. Zhou, W. Qiu, M. Ding, S. Zhang, "Automatic needle segmentation in 3D ultrasound images using 3D Hough transform". SPIE 5th International Symposium on Multispectral Image Processing and Pattern Recognition (2007); p. 67886: 67890

[8] Ding, M., H.N. Cardinal, W. Guan, and A. Fenster, "Automatic needle segmentation in three-dimensional ultrasound images using two orthogonal two-dimensional image projections". Med. Phys. (2003); 2: p. 222: 234.

[9] Aboofazeli, M., P. Abolmaesumi, P. Mousavi, and G. Fichtinger," A new scheme for curved needle segmentation in threedimensional ultrasound images". IEEE Int. Symp. Biomed. Imag.: Nano to Macro (2009); p. 1067: 1070.

[10] A. Ayvaci, P. Yan, S. Xu, S. Soatto, J. Kruecker, "Biopsy needle detection in transrectal ultrasound". Comp. Med. Imag. Graph.(2011); p. 653: 659.

[11] Yue Zhao, Yi Shen, Adeline Bernard, Christian Cachard, Hervé Liebgott, "Evaluation and comparison of current biopsy needle localization and tracking methods using 3D ultrasound". Ultrasonics.(2017); 73: p. 206: 220 .

[12] Parmida Beigi, Pobert Hohling, Tim Salcudean, Victoria A.Lessoway and Gary C.Ng, "Needle trajectory and tip localization in real-time 3-D ultrasound using a moving stylus". Ultrasound in Med. \& Biol. (2015); p. 1: 14.

[13] Irina Rish, "An empirical study of the naive Bayes classifier". workshop on empirical methods in artificial intelligence. (2001); 3 p. 41:46.

[14] A. P.Dempster, N. M.Laird, D. B.Rubin, "Maximum Likelihood from Incomplete Data via the EM Algorithm". Journal of the Royal Statistical Society (1977); 39(1): p. 1: 38 .

[15] Jeff A. Bilmes, "A Gentle Tutorial of the EM Algorithm and its Application to Parameter Estimation for Gaussian Mixture and Hidden Markov Models". Technical Report TR-97-021, International Computer Science Institute (1998).

[16] M. Barva, J. Kybic, J-M. Mari, C. Cahard and V. Hlavac "Automatic Localization of Curvilinear Object in 3D Ultrasound Images". Center for Machine Perception, K13133 FEE Czech Technical University. Prague, Czech Republic, (2007)

[17] Marián Uherčík , Jan Kybic, Herve Liebgott and Christian Cachard, "Model Fitting Using RANSAC for Surgical Tool Localization in 3-D Ultrasound Images". IEEE Transactions on Biomedical Engineering. (2010); 57(8): p. 1907: 1916.

[18] M. A. Fischler and R. C. Bolles, "Random sample consensus: A paradigm for model fitting with applications to image analysis and automated cartography". Communications of the ACM (1981); 24(6): p. 381: 395.

[19] Celine Fouard, Aurélien Deram, Yannick Keraval and Emmanuel Promayon "CamiTK: A Modular Framework Integrating Visualization, Image Processing and Biomechanical Modeling". Stud Mechanobiol Tissue Eng Biomater (2012); 11: p. 323: 354. 